\title{
Broad ligament defects as a cause of chronic pelvic pain
}

\author{
Charlotte Palmer $^{1} \cdot$ David Rowlands $^{1} \cdot$ Vasileios Minas $^{1}$
}

Received: 6 December 2014 / Accepted: 26 May 2015 /Published online: 9 June 2015

(C) Springer-Verlag Berlin Heidelberg 2015

\begin{abstract}
We report a case of a woman with chronic rightsided pelvic pain with an unusual combination of broad ligament defects and associated terminal ileum and caecum herniation. Reduction of the hernia and closure of the defects at laparoscopy resulted in resolution of the patient's painful symptoms. Review of the literature suggests that, although rare, such defects should be considered in the differential diagnosis of persistent pelvic pain. Where found incidentally, broad ligament defects should be repaired in order to prevent sinister complications such as bowel obstruction and strangulation.
\end{abstract}

Keywords Chronic pelvic pain - Broad ligament defect . Laparoscopy $\cdot$ Bowel herniation

\section{Introduction}

Herniation of a viscus through a defect of the broad ligament of the uterus is a rare form of internal hernia [1]. This type of hernia is difficult to diagnose preoperatively due to the non-specific nature of symptoms and limitations of imaging [2]. We report a case of chronic pelvic pain with a diagnosis of bowel herniation through a right-sided broad ligament fenestration and its subsequent laparoscopic management. We review the literature and present our conclusions and recommendations on the diagnosis and management of this condition.

Vasileios Minas

billminas@gmail.com

1 Minimal Access Centre, Department of Obstetrics and Gynaecology, Wirral University Teaching Hospital, Merseyside, UK

\section{Case report}

A 36-year-old woman (one previous vaginal delivery) presented at our outpatient gynaecology clinic with chronic, non-cyclical, intermittent, right-sided pelvic pain and associated bloating. In addition, she reported dysmenorrhoea and occasional dyspareunia.

An initial diagnostic laparoscopy was performed, where the peritoneal defects were seen (right-sided broad ligament fenestration and a fenestration underneath the right uterosacral ligament), but there was no herniation at the time and no corrective measures were taken. Thereafter, a number of medical treatments were tried for her pains including contraceptive pills, the levonorgestrel-releasing intrauterine system and gonadotrophin-releasing analogues with add-back hormone replacement treatment. Whilst these treatments improved her dysmenorrhoea, she still continued to get intermittent pains and bloating. The patient described these pains as non-cyclical, occasionally stabbing and occasionally colicky. The frequency of the pains varied in a given month with several painfree days and several random episodes of pain in a given day, at times with gradual onset, other times with acute onset. A sigmoidoscopy, barium enema and CT scan were performed and showed normal findings.

Three years following her initial presentation, a second laparoscopy was performed due to persistent pains. The caecum and terminal ileum were seen to be herniating through a large (approximately $6 \mathrm{~cm}$ ) right-sided broad ligament fenestration. There was no evidence of bowel ischaemia or necrosis. The hernia was reduced by gently manipulating the bowel, assisted by the Trendelenburg position, and the defects were carefully mapped. A further fenestration was identified underneath a deficient right uterosacral ligament (Fig. 1). The broad ligament defect was closed using a continuous 2-0 Polysorb suture with intra-corporeal knotting. The right uterosacral 
Fig. 1 a Caecum herniating through the right broad ligament defect, b right broad ligament defect as seen after the hernia was reduced, $\mathbf{c}$ right uterosacral ligament fenestration, and $\mathbf{d}$ repair of defect by continuous laparoscopic suture and intracorporeal knotting. $c$ caecum, $s$ uterosacral ligament, $u$ uterus, $r$ round ligament, $f$ fallopian tube, $i$ infundibulopelvic ligament
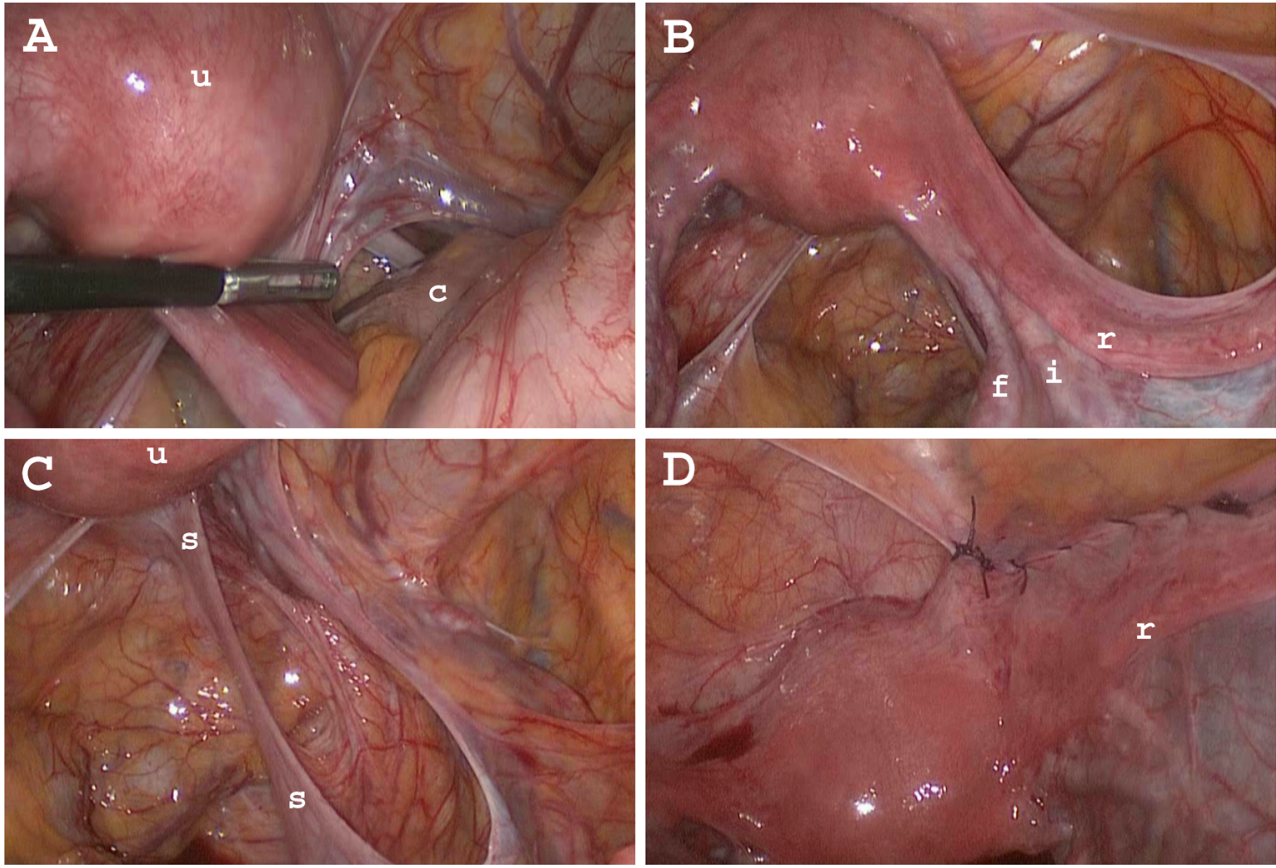

ligament was transected to prevent any future bowel herniation. Repair of this defect by suturing was not deemed sensible as the deficient ligament was too thin. No other pelvic pathology was seen. Six months later, the patient reported complete resolution of her symptoms.

\section{Discussion}

Herein, we present a case of intestinal herniation through a large broad ligament defect, repair of which resolved the chronic painful symptoms of the patient. Such hernias represent 4-7\% of all internal hernias [3]. The most common viscus to herniate is the ileum, however, colonic and ovarian herniation have also been reported [3-5]. Broad ligament defects were first documented in the literature in 1861 following a post-mortem on a woman who died from intestinal obstruction [1]. She was found to have a strangulated bowel in a rightsided broad ligament fenestration.

The precise pathogenesis of broad ligament defects remains unknown. They tend to be unilateral, more commonly on the right side and are thought to be either congenital or acquired [3]. Congenital defects may be related to ruptured cystic structures reminiscent of the mesonephric or paramesonephric ducts during embryological life [3]. Acquired or secondary causes may include previous surgery, previous pregnancy, pelvic inflammatory disease and endometriosis. Preoperative diagnosis is difficult if not impossible. Whilst computed tomography can be useful in identifying acutely dilated bowel in cases where herniation has resulted in bowel obstruction, it is very difficult to pinpoint accurately the broad ligament as the site of internal hernia with incarceration [2].
Two systems for characterising broad ligament defects have been described in the literature. In 1934 Hunt et al. categorised defects based on the nature of the deficiency (complete versus incomplete fenestrations) [6], whereas in 1986, Cilley et al. created a more commonly used classification system based on anatomical position [1]. According to Cilley et al., type I defects are the most common and occur throughout the entire broad ligament caudal to the round ligament. Type II defects occur superior to the round ligament, including defects in the infundibulopelvic ligament, mesosalpinx and ovarian ligament. Type III defects occur in the two layered peritoneal surface between the round ligament and the remainder of the broad ligament. In our case, we diagnosed type II and type III defects with an unusual combination of a defect underneath a thin right uterosacral ligament.

The majority of literature reporting such defects relates to acute presentations of abdominal pain and visceral incarceration, most commonly bowel obstruction [6-10]. Our search returned 39 cases of bowel herniation through broad ligament defects with associated obstruction. The reports date from 1965 to 2014. Although it is beyond the scope of our present paper to cite all these publications, it is interesting to note that in 6 of these cases, preoperative diagnosis was possible by multi-detector computed tomography (MDCT, high resolution computed tomography) [2, 11-15]. Barbier Brion et al. suggest that MDCT can demonstrate the presence and the precise location of this type of hernia and that this may assist clinicians approach the problem laparoscopically rather than by explorative laparotomy [14]. Eight out of the 39 cases were managed by laparoscopy and the rest 31 by laparotomy.

Our case highlights a more chronic course of pelvic pain associated with broad ligament defects. Three published cases 
exist, whereby chronic pain was considered to be associated with broad ligament defects $[3,16,17]$. Redwine reported a case of a woman diagnosed with endometriosis affecting the broad ligament and treated laparoscopically by fulguration [16]. The symptoms of the patient persisted and at repeat laparoscopy the small bowel and the caecum were seen herniating through a broad ligament defect. The defect was repaired and the patient's pains resolved. The author suggested that the defect was a result of the electrocoagulation applied to treat endometriosis. Similarly, Demir and Scoccia described a case of chronic pelvic pain resistant to medical and surgical treatments for endometriosis. A broad ligament defect was diagnosed at laparoscopy and left untreated. At subsequent laparoscopy the ovary was seen to be herniating through the defect. A unilateral salpingo-oophorectomy was performed, the defect was eliminated and the patient's symptoms resolved ${ }^{3}$. Bangari and Uchil reported herniation of small intestine through a large right-sided broad ligament defect, this time with no associated endometriosis, suturing of which cured the patient's chronic pelvic pain [17]. Of note, no complications were reported as a result of repair of broad ligament defects in any of the papers we reviewed describing either acute or chronic presentations.

Similarly, with complete symptom resolution following laparoscopic repair, we believe the most likely cause of our patient's pain was intermittent herniation of bowel through the defect. We therefore strongly support the suggestion by Bangari and Uchil [17] and Demir and Scoccia [3], of repairing or eliminating any incidental broad ligament defects found at laparoscopy. This may not only prove curative for the patient's painful symptoms, but also prevent future sinister complications such as bowel obstruction and strangulation, and/or repeated surgical intervention due to persistent pain.

\section{Conclusion}

Internal hernias through a broad ligament defect, though rare, should be considered in the differential diagnosis of women presenting with chronic pelvic pain, particularly when this is right-sided. This is further relevant in cases where routine investigations have failed to identify pathology and/or routine treatments have failed to relieve symptoms. Laparoscopic management can be both diagnostic and therapeutic, and we recommend that repair by suturing ideally be carried out at initial identification, even if no herniation is seen at the time.

Compliance with ethical standards All procedures followed were in accordance with the ethical standards of the responsible committee on human experimentation (institutional and national) and with the Helsinki Declaration of 1975 , as revised in 2008.

Informed consent was obtained from all patients for being included in the study.
Authors' contribution C. Palmer collected the data and wrote the manuscript. D. Rowlands was responsible for project development. V. Minas also wrote the manuscript and edited it.

Conflict of interest On behalf of all the authors, the corresponding author states that there is no conflict of interest.

\section{References}

1. Cilley R, Poterack K, Lemmer J, Dafoe D (1986) Defects of the broad ligament of the uterus. Am J Gastroenterol 81:389-391

2. Quiroga S, Sarrias M, Sanchez JL, Rivero J (2012) Small bowel obstruction secondary to internal hernia through a defect of the broad ligament: preoperative multi-detector $\mathrm{CT}$ diagnosis. Abdom Imaging 37:1089-1091

3. Demir H, Scoccia B (2010) Internal herniation of adnexa through a defect of the broad ligament: case report and literature review. J Minim Invasive Gynecol 17:110-112

4. Langan RC, Holzman K, Coblentz M (2012) Strangulated hernia through a defect in the broad ligament: a sheep in wolf's clothing. Hernia 16:481-483

5. Lo K, Lie K (2013) Internal herniation through a broad ligament defect found at laparoscopy. J Obstet Gynaecol Can 35:401-402

6. Hunt AB (1934) Fenestra and pouches in the broad ligament as an actual and potential cause of strangulated intra-abdominal hernia. Surg Gynecol Obstet 58:906-913

7. Garcia-Oria M, Inglada J, Domingo J et al (2007) Small bowel obstruction due to broad ligament hernia successfully treated by laparoscopy. J Laparoendosc Adv Surg Tech A 17:666-668

8. Hiraiwa K, Morozumi K, Miyazaki H, Sotome K, Furukawa A, Nakamaru M (2006) Strangulated hernia through a defect of the broad ligament and mobile caecum: a case report. World J Gastroenterol 12:1479-1480

9. Onida S, Lynes K, Ozdemir BA, Whitehouse PA (2010) Unexpected findings at diagnostic laparoscopy: caecal incarceration with concurrent appendicitis in a patient with bilateral broad ligament defects. Ann R Coll Surg Engl 92:W19-20

10. Agresta FM, Nucgeket I, Candiotto E, Bedin N (2007) Incarcerated internal hernia of the small intestine through a breach of the broad ligament: two cases and a literature review. JSLS 11:225-227

11. Kosaka N, Uematsu H, Kimura H, Yamamori S, Hirano K, Itoh H (2007) Utility of multi-detector CT for pre-operative diagnosis of internal hernia through a defect in the broad ligament. Eur Radiol 17:1130-1133

12. Matsunami M, Kusanagi H, Hayashi K, Yamada S, Kano N (2014) Broad ligament hernia successfully treated by laparoscopy: case report and review of literature. Asian J Endosc Surg 7:327-329

13. Chapman VM, Rhea JT, Novelline RA (2003) Internal hernia through a defect in the broad ligament: a rare cause of intestinal obstruction. Emerg Radiol 10:94-95

14. Barbier Brion B, Daragon C, Idelcadi O, Mantion G, Kastler B, Delabrousse E (2011) Small bowel obstruction due to broad ligament hernia: computed tomography findings. Hernia 15:353-355

15. Mailleux P, Ramboux A (2010) Small bowel obstruction due to an internal herniation through a defect of the broad ligament. JBRBTR 93:201-203

16. Redwine DB (1989) Symptomatic internal hernia of the broad ligament: a complication of electrocoagulation of endometriosis. Obstet Gynecol 73(part 2):495-496

17. Bangari R, Uchil D (2012) Laparoscopic management of internal hernia of small intestine through a broad ligament defect. J Minim Invasive Gynecol 19:122-124 